\title{
EXTINCTION MEASUREMENTS WITH LOW-POWER HSRL SYSTEMS—ERROR LIMITS
}

\section{Ed Eloranta}

\section{Affiliation: University of Wisconsin-Madison,US, *eloranta@ssec.wisc.edu}

\begin{abstract}
HSRL measurements of extinction are more difficult than backscatter measurements. This is particularly true for low-power, eye-safe systems. This paper looks at error sources that currently provide an error limit of $\sim 10^{-5} \mathrm{~m}^{-1}$ for boundary layer extinction measurements made with University of Wisconsin HSRL systems. These eye-safe systems typically use $\sim 300 \mathrm{~mW}$ transmitters and $40 \mathrm{~cm}$ diameter receivers with a $10^{-4}$ radian field-of-view.
\end{abstract}

\section{INTRODUCTION}

The HSRL provides both backscatter and extinction cross-sections measurements. The backscatter crosssection measurement is derived from a ratio of signals; calibration is easy and robust. The extinction crosssection measurement is more difficult. It can be derived by taking the $\log$ of the HSRL molecular signal as a function of range, $r$, and then differentiating with respect to $r$ :

$$
\beta_{e}(r)=\frac{1}{2} \cdot \frac{d}{d r}\left[\log \left(\frac{\eta(r) \cdot \beta_{m}(r)}{r^{2} \cdot S_{m}(r)}\right)\right]
$$

This provides the total extinction cross-section, $\beta_{\mathrm{e}}$ as function of the HSRL recovered molecular profile, $S_{\mathrm{m}}$, the overlap correction, $\eta$ and the molecular scattering cross-section, $\beta_{\mathrm{m}} . \quad \beta \mathrm{m}$ is computed from an independent sounding. The extinction due to particulates alone can be derived by simply subtracting the molecular extinction cross-section.

It is instructive to write the equation as follows:

$$
\beta_{e}(r)=\frac{1}{2 \eta} \frac{d \eta}{d r}+\frac{1}{2 \beta_{r}} \frac{d \beta_{m}}{d r}-\frac{1}{r}-\frac{1}{2 S_{m}} \frac{d S_{m}}{d r}
$$

molecular extinction cross-sections in the boundary layer is $\sim 10^{-5} \mathrm{~m}^{-1}$. Aerosol extinction on clear days is often less than the molecular. Thus, it would desirable to keep uncertainty of each term of the equation to less than $10^{-6} \mathrm{~m}^{-1}$. This paper will consider extinction cross-section errors segregated by the terms of equation 2.

\section{OVERLAP CORRECTION}

Figure 1 plots the overlap correction term of equation 2 for one of our lidars. Our low-power, $\sim 300 \mathrm{~mW}$, eyesafe systems employ a $10^{-4}$ radian field-of-view to reduce sky noise. The small field-of-view coupled with a $40 \mathrm{~cm}$ diameter receiver requires overlap corrections for altitudes below $6 \mathrm{~km}$.

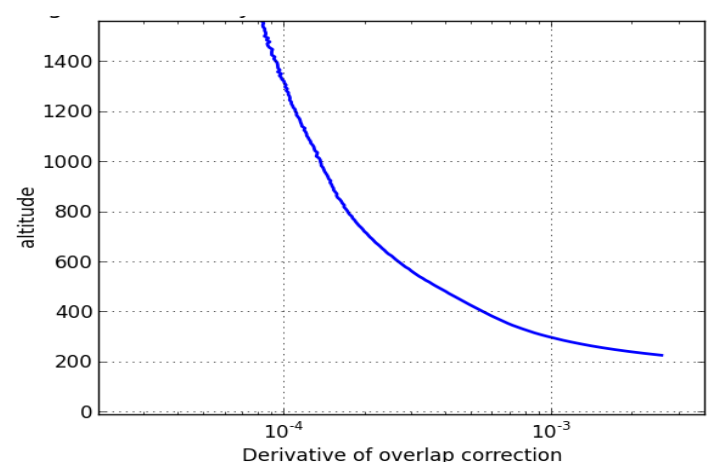

Figure 1. First term of equation 2 for a UW HSRL with a lo0urad field-of-view.

Notice that in order to maintain an overlap correction error in the extinction below $10^{-6} \mathrm{~m}^{-1}$ at $300 \mathrm{~m}$, the overlap correction must be known at the $0.1 \%$ level.

Recent UW HSRL systems include a wide-field-ofview (WFOV) channel to determine $\eta(\mathrm{r})$. This consists of a $2.5-\mathrm{cm}$ diameter telescope mounted behind the secondary mirror of the main telescope. It is coupled via optical fiber to a second molecular HSRL channel consisting of a interference filter, an etalon to block sky background light, and a molecular iodine filter to block aerosol scattering. This channel duplicates the molecular of the HSRL except that it views a $1 \mathrm{mr}$ angular field-of-view rather that the $0.1 \mathrm{mr}$ viewed by the main molecular channel. The WFOV channel provides complete overlap, with $\eta(\mathrm{r})$ equal to 1 , at $\sim 500 \mathrm{~m}$ from the lidar. Figure 2 shows the WFOV-to-molecular channel ratio after it is normalized to one above $6 \mathrm{~km}$. It also shows $\eta(\mathrm{r})$. This is obtained from the ratio of the signals in the WFOV and molecular channels at ranges between $500 \mathrm{~m}$ and $6 \mathrm{~km}$. Generation of the correction factor below $500 \mathrm{~m}$ relies on cases with a well-mixed boundary that extends above $500 \mathrm{~m}$ where we can measure both the 
extinction and the backscatter cross-section accurately. Below $500 \mathrm{~m} \eta(\mathrm{r})$ is determined from the ratio of the expected return from the expected molecular to the measured molecular return. The expected molecular return is computed from the density profile corrected by the extinction computed from the measured backscatter profile and the backscatter phase function measured above $500 \mathrm{~m}$.

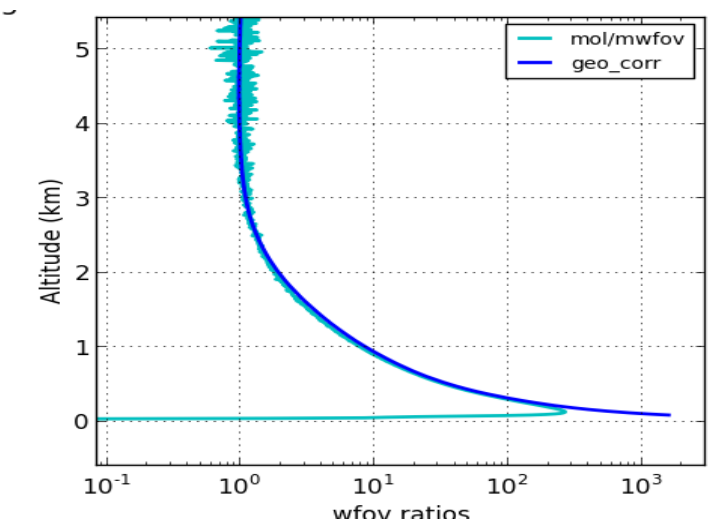

Figure 2. The ratio between the WFOV and the standard molecular channel signals scaled to one above $6 \mathrm{~km}$ (cyan) and $\eta(r)$ (blue). The WFOV/molecular ratio correction is smoothed to generate $\eta(r)$ values at altitudes with full overlap of the WFOV channel. Below full WFOV overlap, $\eta(r)$ is computed using the measured molecular return and the expected atmospheric return. The atmospheric return is computed from the density profile corrected for extinction. Extinction is estimated from the backscatter cross-section and backscatter phase function measured in the mixed layer.

The WFOV channel views a solid angle 100 times as large as the main channel using an objective with 0.0039 times the area. Because it has much poorer noise performance than the molecular channel, overlap corrections must be computed from time averages of the WFOV signal. These overlap corrections provide good correction when generated from, and applied, to night-time data.

Even though the HSRL telescope uses an athermal design, employing carbon fiber construction, and Zerodur optical elements, the system is unable to maintain the necessary stability during mid-day. Overlap variations appear to result from some combination of thermal changes in the transceivertelescope and beam spread caused by atmospheric turbulence. A time-varying overlap correction is required. This is done by observing the ratio of WFOV-to-molecular channel ratio as a function of time and altitude. The overlap correction changes in direct proportion to measured changes in this ratio. These are used to dynamically adjust the overlap correction. In order to achieve adequate signal-to- noise, the ratio is fit with a first- or second-order Savitzky-Golay filter inside a rolling window centered on the observation time. Typical window sizes range from 1 to 2 hours in time and 100-300 meters in altitude. Figure 3 shows a typical example of the backscatter along with the corresponding extinction and the dynamic overlap correction. Plots of extinction and extinction corrections are shown three different altitudes in figure 4. At mid-day (4 UT) the correction was $-8 \times 10^{-5} 1 / \mathrm{m}$.
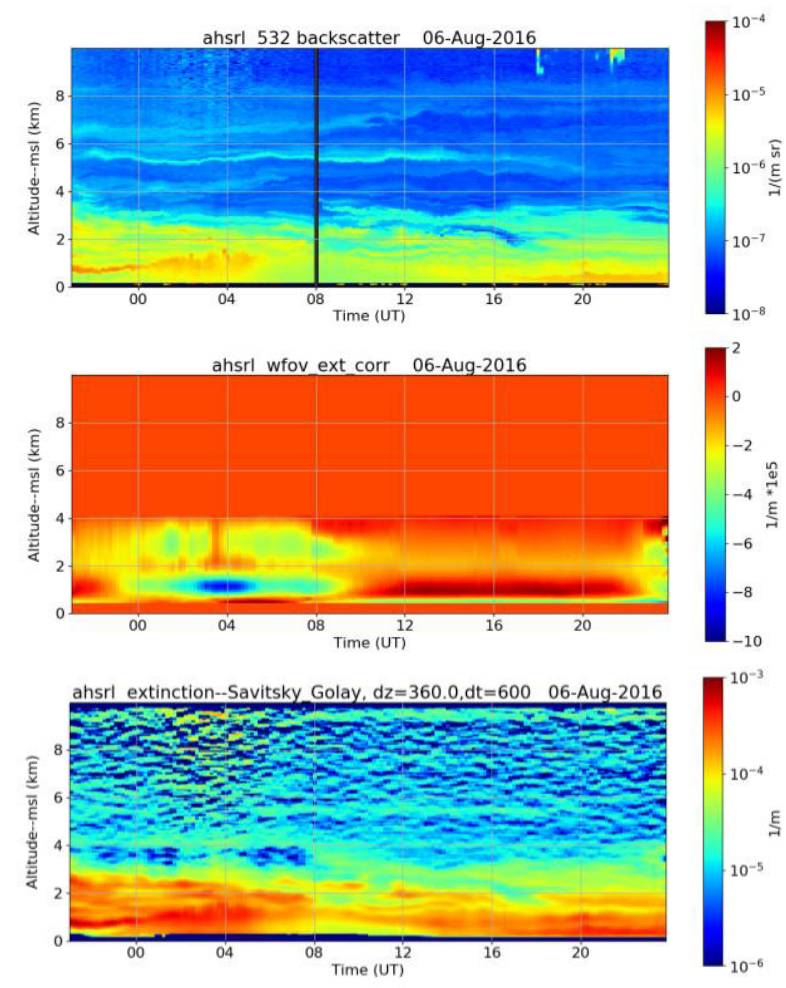

Figure 3. Aerosol Backscatter cross-section (top), dynamic extinction cross-section correction (middle), and extinction cross section (bottom) derived for HSRL data acquired on 6-Aug-16.

\section{TEMPERATURE PROFILE ERRORS}

The second term on the right-hand side of Equation 2 is evaluated from an independently measured temperature profile. The molecular extinction crosssection is directly proportional to the atmospheric density so that for a vertically pointing lidar:

$$
\frac{1}{\beta_{m}} \frac{d \beta_{m}}{d z}=\frac{1}{\rho} \frac{d \rho}{d z}=-\frac{1}{T}\left(\frac{d T}{d z}-\frac{g}{R}\right)
$$

Where: $\rho=$ density, $\mathrm{g}=$ acceleration of gravity, $\mathrm{z}=$ altitude, and $\mathrm{R}$ $=$ gas constant. 

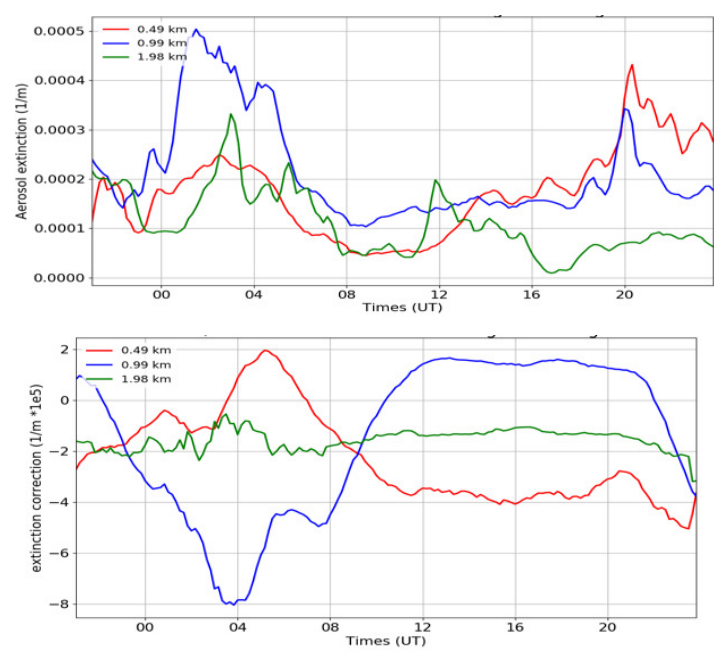

Figure 4. Derived extinction cross-section(upper) and corrections due variations in first term of equation 3 for the data shown in figure 3. Curves are shown for altitudes of 490 (red), 990(blue) and 1990(green) meters.

The temperature resolution to achieve a given $\beta_{\mathrm{e}}$ error can be obtained by differentiating Equation 3 .

$$
\Delta \beta_{e}=\frac{1}{T} \cdot\left(\frac{d T}{d z}+\frac{g}{R}\right) \cdot \Delta T+\frac{1}{T} \cdot \Delta \frac{d T}{d z}
$$

A $2 \mathrm{~K}$ temperature error at $300 \mathrm{~K}$, and a lapse rate of $6 \mathrm{~K} / \mathrm{km}$ produces a only $\sim 10^{-6} \mathrm{~m}^{-1}$ error from the first term of equation 4. Lapse rate errors are more significant. Near surface temperature profiles transition from inversion to adiabatic lapse rates on most summer mornings providing extinction variations:

$$
\frac{1}{T} \Delta \frac{d T}{d z} \sim \frac{1}{300} \cdot \frac{10}{1000} \sim 3 \cdot 10^{-5} m^{-1}
$$

Figure 5 shows lapse rates derived from soundings taken at 6-hour intervals in Seoul, South Korea. Notice the low altitude transitions between inversion and lapse conditions. Their effect is clearly seen in the $0.53 \mathrm{~km}$ time history of HSRL extinction. At 16:00 UT the extinction suddenly decreases by nearly $10^{-5} \mathrm{~m}^{-1}$ as the lapse rate transitions from lapse to inversion and then increases abruptly by nearly $2 \times 10^{-5} \mathrm{~m}^{-1}$ at $\sim 1: 00$ UT when the inversion conditions switch back to lapse. Between these transitions the derived extinction goes negative. This most likely due to the complex terrain of
Seoul reflecting a difference between atmospheric conditions at the radiosonde site and the lidar site.
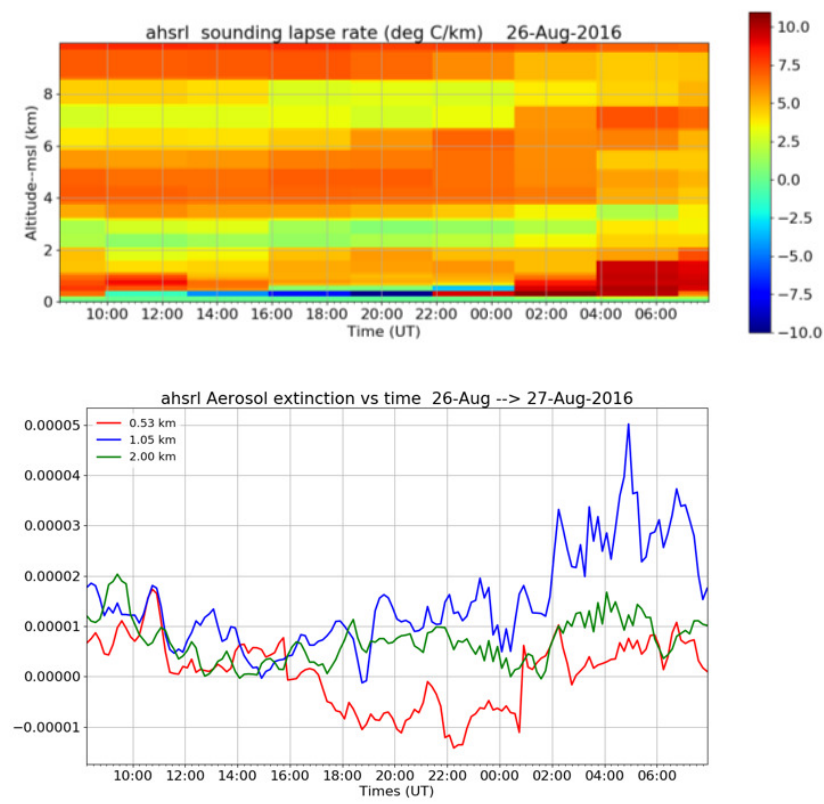

Figure 5. Lapse rate vs time and altitude (top) and derived extinction cross-sections at altitudes of $0.53 \mathrm{~km}$ (red), $1.05 \mathrm{~km}$ (blue) and $2.0 \mathrm{~km}$ (green). Notice the jumps in extinction at $0.53 \mathrm{~km}$ occurring at 16:00 and 1:00 UT. These correspond to changes from inversion to lapse in the lapse rate plot.

\section{PHOTON COUNTING STATISTICS}

We now consider the final term of Equation 2. The molecular profile, $S_{m}$, is derived from the number of photons detected in the HSRL molecular, $\mathrm{N}_{\mathrm{m}}$, and combined, $\mathrm{N}_{\mathrm{c}}$, channels of the instrument:

$S_{m}(r)=k(r) \cdot\left(N_{m}(r)-C_{a m} N_{c}(r)^{\prime}\right.$,

Outside of clouds $\mathrm{N}_{\mathrm{m}}$ and $\mathrm{N}_{\mathrm{c}}$ are of similar magnitude, while $\mathrm{C}_{\mathrm{am}}<0.0005$, the leakage of aerosol signal into the molecular channel, can be neglected outside of clouds. The last term of Equation 2 can then be written as:

$\frac{1}{S_{m}(r)} \frac{d S_{m}}{d r}=\frac{1}{k(r)} \frac{d k(r)}{d r}+\frac{1}{N_{m}(r)} \frac{d\left(N_{m}(r)+N_{b}\right)}{d r}$

Errors in the logarithmic range derivative of the molecular signal are dependent on how accurately the HSRL calibration separates molecular and particulate signals as well as statistical photon counting errors. Notice that the range derivative now also includes the background counts, $\mathrm{N}_{\mathrm{b}}$, from sky and detector noise. Although the average number of background counts does not vary with range, counting errors will appear as range variations. The first term on the right hand side includes the range variation of the calibration coefficients used to separate molecular and particulate 
photons. These coefficients are determined through calibration of the spectral bandpass of the receiver channels and knowledge of the atmospheric temperature profile. They are quite well measured and are almost always dominated by photon counting error.

Photon counting errors are described via Poisson statistics where the signal variance is equal to the number of photon counts. The problem posed by the $\mathrm{N}_{\mathrm{m}}$ term can be seen by approximating the error for best case where the molecular photon counting rate is near the point of detector saturation. The Geiger-mode avalanche photodiode detectors used in our HSRL systems are unable to count more than $\sim 1$ photon per $7.5 \mathrm{~m}$ range bin. With the system operating at a $4 \mathrm{kHz}$ repetition rate and a 2.5 second averaging time, a single range bin can count a maximum of $10^{4}$ photons in one sample. Approximating the derivative from the counting error for 2.5 -second profiles and a separation of one range bin shows that even in the case of strong signals, photon counting statistics can provide a large source of error.

$$
\frac{1}{N_{m}(r)} \frac{d N_{m}(r)}{d r} \sim \frac{1}{10^{4}} \frac{\sqrt{2} \cdot \sqrt{10^{4}}}{7.5} \sim 2 \cdot 10^{-3} \mathrm{~m}^{-1}
$$

This error is approximately three orders of magnitude larger than the $10^{-6} \mathrm{~m}^{-1}$ extinction cross-section we might like to measure. Clearly, it will be necessary to average over longer time and space intervals to reduce errors near those that are desired.

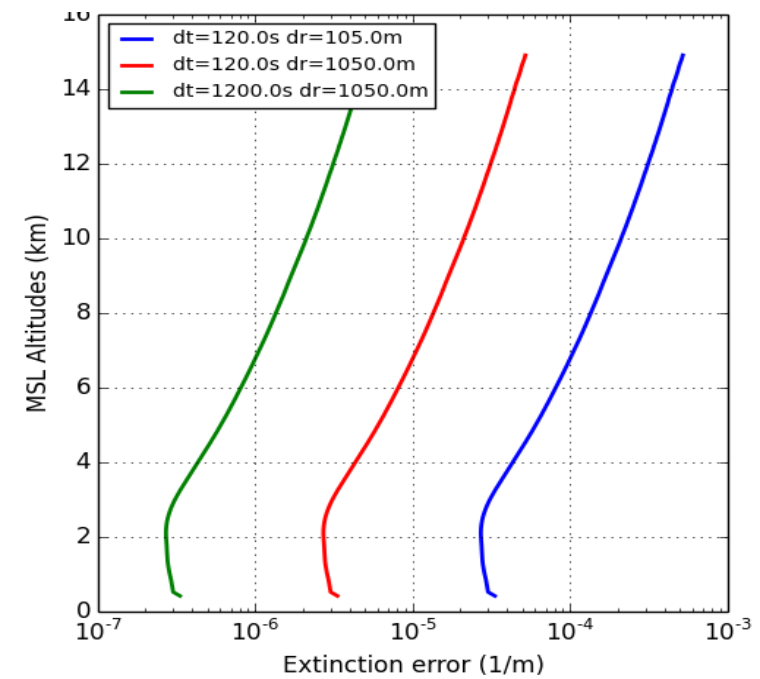

Figure 6. Extinction errors due to photon counting statistics for different time and range averages estimated from the last term on the left-hand side of Equation 2. These results computed from clear-air returns on 2-June-2012.

Figure 6 shows that photon counting errors in the extinction measurement can be reduced below $10^{-6} \mathrm{~m}^{-1}$ below $7 \mathrm{~km}$ using 20-minute time and $1 \mathrm{~km}$ range averaging. This amount of averaging may be practical in stable cloud free conditions, but may obscure the temporal and spatial scales of the atmospheric structures in more variable conditions. Typical time averages $\sim 20$ minutes and altitude averages of $\sim 200 \mathrm{~m}$ provide a photon counting error limit below $10^{-5} \mathrm{~m}^{-1}$ with our current systems.

\section{CONCLUSIONS}

Although extinction cross-section is a more useful quantity than backscatter cross-section, it is more difficult to measure. With current hardware and algorithms boundary layer measurement errors of $\sim 10$ $5 \mathrm{~m}^{-1}$ can often be obtained with current UW HSRL system using $\sim 300 \mathrm{~mW}$ transmitters and $10^{-4} \mathrm{mr}$ fieldof-view receivers. Limits are imposed by photon counting noise along with imprecise knowledge of the overlap function and the atmospheric density profile. We are working to derive temperature profiles from lidar data and thus reduce uncertainties due to temperature.

\section{ACKNOWLEDGEMENTS}

This research was supported by US Department of Energy contract 258032.

\section{References}

[1] Eloranta, E.W., "High Spectral Resolution Lidar", in Lidar: Range-Resolved Optical Remote Sensing of the Atmosphere, K. Weitkamp editor, SpringerVerlag, New York, 2005.

[2] University of Wisconsin lidar group web site: $\underline{\text { lidar.ssec.wisc.edu }}$

[3] Savitzky, A; Golay, M.J.E. (1964), "Smoothing and Differentiation of Data by Simplified Least Squares procedure", Analytical Chemistry, 36, (8), 1627-163 